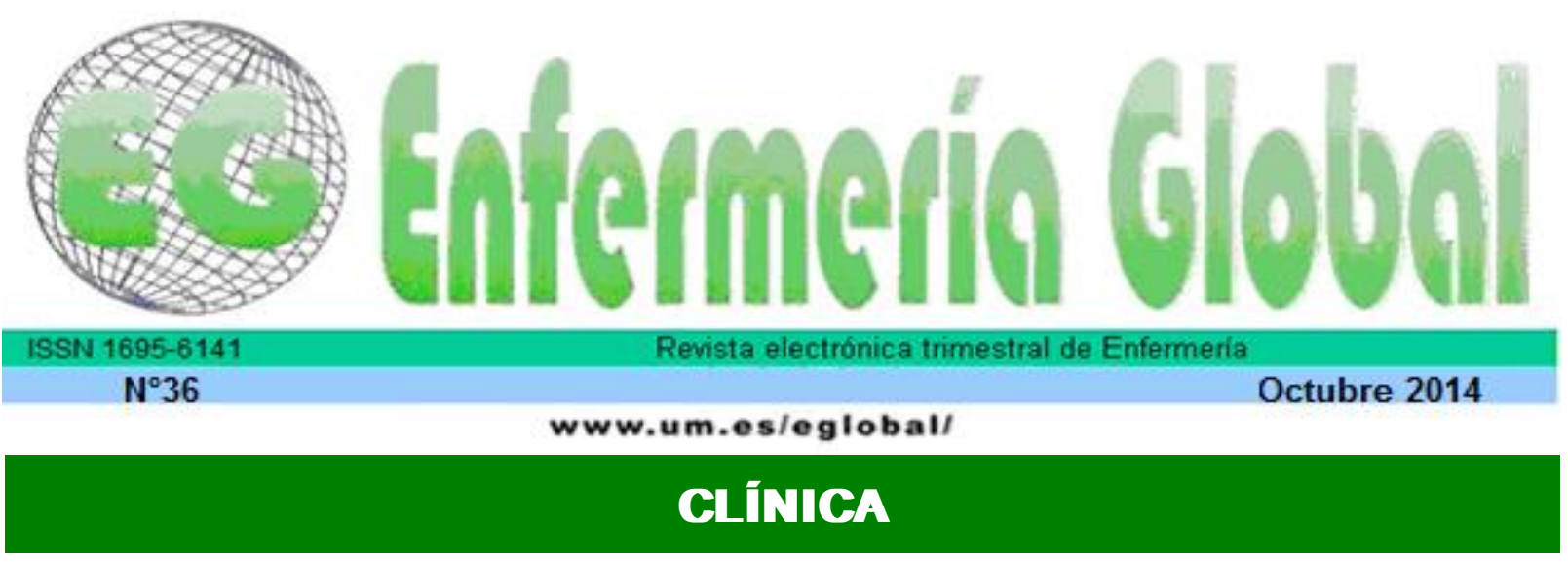

\title{
Compromiso tisular de pacientes con úlcera venosa según las clasificaciones de los resultados de enfermería
}

Comprometimento tissular de pacientes com úlcera venosa de acordo com a classificação dos resultados de enfermagem

Impairment of patients with venous ulcers according to the outcome nursing classification

\section{*Almeida Medeiros, Ana Beatriz *Farias de Queiroz Frazão, Cecília Maria ${ }^{* *}$ Dantas de Sá, Jéssica ${ }^{* * *}$ Brandão de Carvalho Lira, Ana Luisa ${ }^{* * * *}$ Venícios de Oliveira Lopes, Marcos}

*Máster en Enfermería. Departamento de Enfermería, Universidad Federal de Rio Grande do Norte, Natal. E-mail: abamedeiros@gmail.com **Estudiante de Enfermería de la Universidad Federal de Rio Grande do Norte, Natal, en movilidad académica en la Universidad Católica San Antonio de Murcia, España. ${ }^{* * *}$ Doctora en Enfermería. Departamento de Enfermería, Universidad Federal de Rio Grande do Norte, Natal. ${ }^{* * * *}$ Doctor en Enfermería. Departamento de Enfermería, Universidad Federal de Ceará, Fortaleza, Brasil.

(Estudio financiado por el Programa de apoyo a planes de Reestructuración y Expansión de las Universidades Federales - REUNI)

\section{Palabras clave: Úlcera venosa; Enfermería; Clasificación; Evaluación de resultado Palavras chave: Úlcera venosa; Enfermagem; Classificação; Avaliação de resultados Keywords: Venous ulcer; Nursing; Classification; Outcome assessment}

\section{RESUMEN}

La ulcera venosa es un problema epidemiológico de alta prevalencia que provoca incapacidad y dependencia. Evaluar el compromiso tisular de pacientes con lesiones venosas, basado en un referencial específico de Enfermería, puede direccionar los cuidados de acuerdo con las especificidades de los pacientes.

Se desarrolló un estudio transversal con objetivo de identificar el grado de compromiso de la integridad tisular de pacientes con ulcera venosa de acuerdo con la clasificación de los resultados de enfermería.

La investigación se llevó a cabo en un hospital universitario brasileño, con 50 pacientes, elegidos por muestreo consecutivo. Se utilizó un formulario compuesto por 18 indicadores, aplicado en los meses de febrero a junio de 2012. Se utilizó la estadística descriptiva para el análisis de los datos. Cinco 
indicadores presentaron comprometimiento moderado (mediana $=3$ ): dolor, perfusión tisular, pigmentación anormal, crecimiento del vello cutáneo y tejido cicatricial.

Se concluyó que la mayoría de los indicadores evaluados en pacientes entrevistados presentó grado de compromiso variando desde leve hasta moderado.

\section{RESUMO}

A úlcera venosa é um problema epidemiológico de alta prevalência, que provoca incapacidade e dependência. Avaliar o comprometimento tissular de pacientes com lesões venosas, dentro de um referencial próprio da Enfermagem, pode direcionar os cuidados conforme as especificidades dos pacientes.

Tratou-se de um estudo transversal, com o objetivo de identificar o grau de comprometimento da integridade tissular de pacientes com úlcera venosa de acordo com a classificação dos resultados de enfermagem.

A pesquisa foi realizada em um hospital universitário brasileiro, com 50 pacientes, selecionados através de amostragem consecutiva. Utilizou-se um formulário constituído por 18 indicadores, aplicado nos meses de fevereiro a junho de 2012. Utilizou-se a estatística descritiva para a análise dos dados. Cinco indicadores apresentaram comprometimento moderado (mediana $=3$ ): dor, perfusão tissular, pigmentação anormal, crescimento cutâneo de pelos e tecido cicatricial.

Conclui-se que a maioria dos indicadores avaliados nos pacientes entrevistados apresentou grau de comprometimento variando de leve a moderado.

\section{ABSTRACT}

The venous ulcer is an epidemiological problem of high prevalence, causing disability and dependence. Evaluate the tissue impairment of patients with venous lesions, within a nursing referential can directed the care of patients as the specifics.

It was a cross-sectional study, aiming to identify the level of impairment tissue integrity of patients with venous ulcers according to the classification of nursing outcomes.

The research was conducted at a brazilian university hospital with 50 patients, selected by sampling consecutive. We used a form consists of 18 indicators, applied from February to June 2012. Data analysis was done by descriptive statistics. Five indicators showed moderate impairment (median $=3$ ): pain, tissue perfusion, defective pigmentation, skin hair growth and cicatricial tissue.

It was oncluded that most indicators evaluated in the patients interviewed had impairment, varying from mild to moderate.

\section{INTRODUCCIÓN}

La úlcera venosa (UV) es una manifestación clínica grave de la insuficiencia venosa crónica (IVC), derivada de la hipertensión venosa de larga duración relacionada con la insuficiencia valvular y/u obstrucción venosa. Las UV se caracterizan por pérdida de sustancia de la epidermis/dermis y afectan a las extremidades de los miembros inferiores (MMII), más frecuentemente en la región maleolar, representando aproximadamente entre $70 \%$ y $90 \%$ de los casos de úlceras de las piernas ${ }^{(1-4)}$.

El miembro afectado presenta características como venas varicosas, telangiectasia, venas reticulares, dolor, edema, prurito, eccema de estasis, lipodermatoesclerosis, hiperpigmentación y atrofia blanca. Cuando espontáneas, las úlceras suelen estar situadas justo por encima de los maléolos, sobre una vena insuficiente donde hay gran hipertensión ${ }^{(2,5)}$. 
Las características de las úlceras venosas incluyen: formato irregular, inicialmente superficial, pero con potencial de profundización, situado en el centro de la placa endurecida, eritematosa, escamosa o hiperpigmentada. Su aspecto es variable, extendiéndose sobre grandes áreas, con presencia de exudado serosanguinolento (en la presencia de la granulación) o seropurulento (en los casos de infección secundaria). Además, se presenta en forma de islas de epitelización ${ }^{(1,5,6)}$.

El gran cuantitativo de recidiva de las UV constituye uno de los problemas más importantes en el cuidado de los pacientes portadores de insuficiencia venosa. Por lo tanto, la caracterización del estado de salud del paciente se convierte en una prioridad en la atención de enfermería ${ }^{(3)}$. Para que el enfermero pueda caracterizar el estado de salud de estos pacientes adecuadamente es interesante hacer uso de instrumentos de evaluación específicos. Para ello, la presente investigación utilizó la Clasificación de los Resultados de Enfermería (Nursing Outcomes Classification NOC) que, además de conceptualizar, definir y clasificar los resultados e indicadores sensibles en la atención de enfermería, sirve como medida basal del nivel del estado de salud y evalúa los resultados con base en las intervenciones de enfermería ${ }^{(7)}$. El término "Indicadores de los resultados de enfermería" caracteriza el estado de salud a un nivel concreto ${ }^{(8)}$.

En este estudio se eligió el resultado denominado "Integridad tisular: piel y membranas mucosas", que es definido en la NOC como la integridad estructural y función fisiológica normal de la piel y mucosas ${ }^{(9)}$. Este resultado presenta 21 indicadores y su evaluación está basada en una escala de Likert con cinco niveles que varía desde gravemente comprometido hasta no comprometido. Es importante destacar que una de las limitaciones del uso de la NOC es la ausencia de definiciones operacionales para los niveles de las escalas.

Aunque existan instrumentos de evaluación para evaluación de la piel y de las úlceras por presión, pocos son los instrumentos desarrollados específicamente para evaluar el compromiso tisular de pacientes con úlcera venosa. Por lo tanto, el uso de escalas NOC puede contribuir a un conocimiento específico de los indicadores clínicos que presentan compromiso más evidente, además de contribuir a la normalización del lenguaje enfermero. De ahí que este estudio tuvo como objetivo identificar el grado de compromiso de la integridad tisular de pacientes con úlcera venosa de acuerdo con la clasificación de los resultados de enfermería.

\section{MATERIAL Y MÉTODO}

Estudio transversal, desarrollado en un ambulatorio de clínica quirúrgica de un hospital universitario ubicado en el nordeste de Brasil, durante los meses de febrero a junio de 2012. La población se constituyó por todos los pacientes adultos con UV en tratamiento en el ambulatorio del hospital. La muestra estuvo compuesta por 50 individuos, elegidos por muestreo consecutivo. El tamaño de la muestra ha sido calculado a partir de la fórmula $n=\left(z^{*} s / e\right)^{2}$, donde " $z$ " es el nivel de confianza, "s" es la desviación estándar de la media de la escala NOC y "e", el error absoluto con respecto a la media de la escala $\mathrm{NOC}^{(11)}$. Los parámetros utilizados han sido un nivel de confianza del 95\% ( $z=1,96)$, una desviación estándar de las puntuaciones de la escala NOC de 3 y un error 0,83 punto.

Se incluyeron pacientes con úlcera venosa, diagnosticada por signos clínicos y valor del índice tobillo/brazo (ITB) superior a 0,8, que estaban en seguimiento en el 
ambulatorio de clínica quirúrgica del hospital y con edad por encima de los 18 años. Se excluyeron pacientes con trastornos psiquiátricos o psíquicos, o que presentaran herida oncológica, arterial o de etiología mixta.

Para la recolección de los datos se utilizó un formulario conteniendo datos demográficos y los indicadores del resultado "Integridad tisular: piel y membranas mucosas". Definiciones operacionales desarrolladas y validadas en estudio anterior han sido utilizadas para la evaluación de los indicadores del resultado NOC ${ }^{(10)}$. Los indicadores evaluados han sido: temperatura de la piel, sensibilidad, elasticidad, hidratación, textura, grosor, perfusión tisular, crecimiento del vello cutáneo, pigmentación anormal, lesiones cutáneas, tejido cicatricial, descamación cutánea, eritema, necrosis, induración, prurito, dolor y exudado.

Para la medición de estos indicadores, se hizo uso de los métodos propedéuticos de inspección y palpación. Fueron utilizados dos instrumentos específicos en el examen físico, a saber: termómetro de infrarrojo MT-305 de marca Minipa®, calibrado con la emisividad de 0.98, específico para la piel humana, y los monofilamentos de SemmesWeinstein de la marca Sorri®

El análisis de los datos se hizo por medio de estadísticas descriptivas, incluyendo frecuencias absolutas y relativas, medianas y rangos intercuartílicos. Los datos han sido recogidos tras el consentimiento informado del paciente, respetándose el anonimato y la confidencialidad. El estudio fue aprobado por el Comité de Ética en Investigación de la institución (protocolo 608/11 y certificado de presentación para apreciación ética 0038.0.294.000-11).

\section{RESULTADOS}

Los pacientes tenían edad media de 59,72 años $( \pm 12,57)$, tiempo medio de estudio de 4,98 años $( \pm 3,32), 66 \%$ eran mujeres y un $48 \%$ eran jubilados. La tabla I presenta las medidas descriptivas de los indicadores evaluados.

Tabla I - Caracterización de los pacientes con úlceras venosas en cuanto al deterioro de los indicadores del resultado Integridad tisular: piel y membranas mucosas.

\begin{tabular}{|c|c|c|c|c|c|c|c|c|c|c|c|}
\hline \multirow{2}{*}{ Indicadores } & \multicolumn{2}{|c|}{ Grave } & \multicolumn{2}{|c|}{ Substancial } & \multicolumn{2}{|c|}{ Moderado } & \multicolumn{2}{|c|}{ Leve } & \multicolumn{2}{|c|}{ Ninguno } & \multirow{2}{*}{$\begin{array}{l}\text { Mediana: } \\
\text { RI }\end{array}$} \\
\hline & $\mathbf{N}$ & $\%$ & $\mathbf{n}$ & $\%$ & $\mathbf{n}$ & $\%$ & $\mathbf{n}$ & $\%$ & $\mathbf{n}$ & $\%$ & \\
\hline Dolor & 3 & 6,0 & 16 & 32,0 & 20 & 40,0 & 5 & 10,0 & 6 & 12,0 & $\begin{array}{l}\text { Med = 3: } \\
\mathrm{RI}=1\end{array}$ \\
\hline Perfusión Tisular & 2 & 4,0 & 5 & 10,0 & 31 & 62,0 & 6 & 12,0 & 6 & 12,0 & $\begin{array}{l}\text { Med = } \\
R I=0\end{array}$ \\
\hline $\begin{array}{l}\text { Pigm } \\
\text { anorr }\end{array}$ & 6 & 12,0 & 14 & 28,0 & 12 & 24,0 & 17 & 34,0 & 1 & 2,0 & $\begin{array}{l}\text { Med }=3 \\
R I=2\end{array}$ \\
\hline $\begin{array}{l}\text { Crecimiento } \\
\text { vello cutáneo }\end{array}$ & 6 & 12,0 & 11 & 22,0 & 14 & 28,0 & 19 & 38,0 & - & - & $\begin{array}{l}\text { Med }=3 \\
\mathrm{RI}=2\end{array}$ \\
\hline Tejid & - & - & 7 & 14,0 & 28 & 56,0 & 15 & 30,0 & - & - & $\mathrm{RI}$ \\
\hline Indur & 2 & 4,0 & 3 & 6,0 & 17 & 34,0 & 19 & 38,0 & 9 & 18,0 & $\begin{array}{l}\mathrm{Me} \\
\mathrm{RI}\end{array}$ \\
\hline Erit & - & - & 4 & 8,0 & 12 & 24,0 & 28 & 56,0 & 6 & 12,0 & 1 \\
\hline Grosor & 1 & 2,0 & - & - & 7 & 14,0 & 42 & 84,0 & & - & Med $=\angle$ \\
\hline
\end{tabular}




\begin{tabular}{|c|c|c|c|c|c|c|c|c|c|c|c|}
\hline Exudado & 8 & 16,0 & 1 & 2,0 & 16 & 32,0 & 21 & 42,0 & 4 & 8,0 & $\begin{array}{l}\mathrm{Rl}=0 \\
\mathrm{Med}=4: \\
\mathrm{RI}=1\end{array}$ \\
\hline Hidratación & 1 & 2,0 & 1 & 2,0 & 7 & 14,0 & 29 & 58,0 & 12 & 24,0 & $\begin{array}{l}\text { Med }=4: \\
\mathrm{RI}=0\end{array}$ \\
\hline Prurito & - & - & 6 & 12,0 & - & - & 28 & 56,0 & 16 & 32,0 & $\begin{array}{l}\text { Med }=4 \\
\mathrm{RI}=1\end{array}$ \\
\hline Textura & 2 & 4,0 & 9 & 18,0 & 8 & 16,0 & 29 & 58,0 & 2 & 4,0 & $\begin{array}{l}\text { Med = 4: } \\
\mathrm{Rl}=1\end{array}$ \\
\hline $\begin{array}{l}\text { Descamación } \\
\text { cutánea }\end{array}$ & - & - & - & & 3 & 6,0 & 21 & 42,0 & 26 & 52,0 & $\begin{array}{l}\text { Med = 5: } \\
\mathrm{RI}=1\end{array}$ \\
\hline Elasticidad & 1 & 2,0 & 2 & 4,0 & 3 & 6,0 & 18 & 36,0 & 26 & 52,0 & $\begin{array}{l}\text { Med = } 5 \\
\mathrm{RI}=1\end{array}$ \\
\hline Lesiones cutáneas & 11 & 22,0 & - & - & 2 & 4,0 & 10 & 20,0 & 27 & 54,0 & $\begin{array}{l}\text { Med }=5 \\
\mathrm{RI}=2\end{array}$ \\
\hline Necrosis & 1 & 2,0 & - & - & 4 & 8,0 & 5 & 10,0 & 40 & 80,0 & $\begin{array}{l}\text { Med }=5: \\
\mathrm{RI}=0\end{array}$ \\
\hline Sensibilidad & 2 & 4,0 & - & - & - & 2,0 & 4 & 16,0 & 30 & 60,0 & $\begin{array}{l}\text { Med = 5: } \\
\mathrm{RI}=1\end{array}$ \\
\hline $\begin{array}{l}\text { Temperatura de la } \\
\text { piel }\end{array}$ & 15 & 30,0 & 2 & 4,0 & 1 & 2,0 & - & - & 32 & 64,0 & $\begin{array}{l}\text { Med = 5: } \\
\mathrm{RI}=4\end{array}$ \\
\hline
\end{tabular}

Mediana (Med); (RI) - Rango Intercuartil.

Cinco indicadores presentaron compromiso moderado (mediana $=3$ ): dolor, perfusión tisular, pigmentación anormal, crecimiento del vello cutáneo y tejido cicatricial. El compromiso moderado para el indicador dolor fue evidenciado por el relato de sensación dolorosa por más de una vez a la semana, o dolor al caminar y/o en posición ortostática. La perfusión tisular presentó un compromiso moderado caracterizado por la presencia de venas varicosas.

La pigmentación anormal el nivel de compromiso observado se caracterizó por coloración azul-marrón del área perilesional y de la extremidad afectada. El indicador crecimiento del vello cutáneo se evidenció por el enflaquecimiento del vello alrededor de la úlcera y en área adyacente a la herida. Para el tejido cicatricial, el compromiso moderado se caracterizó por la presencia de tejido de granulación con exudación y ausencia de tejidos con necrosis coagulativa o licuefactiva.

Siete indicadores presentaron un nivel levemente comprometido (mediana 4): induración, eritema, grosor, exudado, hidratación, prurito y textura. El compromiso leve para la induración se caracterizó por el signo de Godet presente con retorno hasta 1 segundo. Para el indicador eritema, dicho compromiso se evidenció por el color rosa claro. La espesura se caracterizó por la exposición de la dermis sin afectar el tejido subcutáneo. El indicador exudado se caracterizó por la presencia de exudado seroso.

El indicador hidratación presentó variación clínica desde de los extremos de la xerosis y maceración hasta un compromiso leve con la presencia de una piel seca alrededor de la lesión. Los indicadores prurito y textura también presentaron variabilidad entre los extremos de la escala NOC. 
La clasificación de no compromiso, referente al valor 5 en la escala Likert, puede ser visualizada en la mediana de seis indicadores, que fueron: descamación cutánea, elasticidad, lesiones cutáneas, necrosis, sensibilidad y temperatura de la piel.

\section{DISCUSIÓN}

El compromiso moderado con relación al indicador dolor está probablemente relacionado con un aumento en la presión venosa o insuficiencia de las válvulas venosas y constituye una influencia negativa en la vida diaria de estos pacientes ${ }^{(4,12-}$ 13,15-16). Los datos identificados en el presente estudio son conducentes con una revisión integradora, que evaluó los impactos que el dolor en la UV desencadena en la vida de las personas ${ }^{(17)}$.

El compromiso moderado del indicador perfusión tisular se justifica por la congestión de flujo sanguíneo, debido a la incompetencia de las válvulas venosas, las venas se caracterizan por una dilatación mayor o igual a $3 \mathrm{~mm}$ de diámetro, medida en la posición vertical y por ser tortuosas ${ }^{(2-4)}$. La proporción de pacientes identificados en el presente estudio es similar a los porcentuales de un estudio de caracterización de los clientes con UV, lo que indica la presencia de venas varicosas en un $76,4 \%$ de los pacientes ${ }^{(12)}$ y con estudio de caracterización de pacientes con UV basado en la Clasificación de los Resultados de Enfermería ${ }^{(8)}$.

La coloración azul-marrón identificada en la mediana de los pacientes está relacionada con el oscurecimiento del pigmento, o hiperpigmentación de la piel, y se produce debido a la acumulación de hemosiderina, que es una consecuencia de la degradación de la hemoglobina extravasada en el intersticio y liberada tras la ruptura de las células rojas ${ }^{(2-5)}$. Estos datos corroboran con los datos de investigación que también hizo uso de los indicadores de la NOC para caracterizar a los pacientes con $\mathrm{UV}^{(8)}$.

El compromiso moderado del crecimiento del vello cutáneo se caracterizó por la rarefacción de vellos alrededor de la úlcera, lo que puede justificarse por el hecho de que la presencia de lesiones crónicas modifican las características normales de la piel. La disminución en la distribución de vellos puede encontrarse alterada debido a la atrofia de la piel, provocada por un proceso inflamatorio crónico, ya que los folículos pilosos se alojan en el tejido celular subcutáneo ${ }^{(13)}$.

Los datos relacionados al indicador tejido cicatricial son consonantes con un estudio sobre evaluación clínica en pacientes ancianos con úlcera en la pierna ${ }^{(14)}$. En el referido estudio los ancianos no presentaron necrosis en las lesiones lo que influyó en la conclusión por un nivel moderado de compromiso similar al presente estudio.

Las úlceras venosas tienen un desarrollo rápido en el inicio y resultan de una lesión que puede ser necrótica o amarillenta y fibrosa dentro de la cual se pueden observar las áreas de granulación de coloración rojiza. De ahí que la cantidad de exudado es variable y depende de la extensión del edema y del nivel de comprometimiento del tejido $^{(15)}$.

La induración presenta relación directa con el edema y es uno de los síntomas más comunes de la ICV. Se caracteriza por un incremento en el volumen de fluido de la piel y del tejido subcutáneo. Esto ocurre debido al hecho de que durante la relajación muscular, la hipertensión venosa aumenta como resultado del reflujo venoso, que 
hace que la presión hidrostática dentro de las venas, vénulas y capilares venosos alcance un valor alto, un fenómeno que provoca la salida de líquido para el espacio intersticial y que se comprueba por la formación del signo de Godet visible a la compresión digital $^{(2-4,13)}$.

El signo de Godet presente con retorno en hasta 1 segundo, visualizada en gran parte de la muestra investigada, muestra que los participantes no tenían un edema tan significativo, que puede justificarse por el uso de métodos compresivos como vendajes de compresión, botas de Unna y calcetines elásticos. La compresión aumenta la presión del tejido, favoreciendo la reducción del reflujo venoso y del edema de la pierna. El reposo y elevación de los miembros inferiores también actúan para disminuir el efecto de la hipertensión venosa ${ }^{(6,17)}$. Estos datos son conducentes con un estudio de adultos con UV, que mostraron un predominio del signo de Godet de $+1 /+4$, considerando una escala de cinco puntos ( $\sin$ edema, $+1 /+4,+2 /+4,+3 /+4$ y $+4 /+4)^{(14)}$.

Eritema significa una coloración roja de la piel y puede indicar una mayor cantidad de sangre en la red vascular cutánea debido a la inflamación y congestión de capilares superficiales. El enrojecimiento o eritema acompaña el proceso inflamatorio como uno de los cuatro signos de inflamación ${ }^{(13)}$. En el presente estudio, el compromiso leve para este indicador equivale a la coloración rosa claro alrededor de la lesión.

El indicador grosor se caracterizó con compromiso leve, especificado como exposición de la dermis sin afectar el tejido subcutáneo, lo que puede explicarse por ser la superficialidad una característica común de este tipo de lesión cutánea ${ }^{(4,6)}$. Esta característica también se encontró en un estudio realizado con ancianos con úlceras en las piernas, el cual identificó un $64,2 \%$ de los participantes con pérdida de tejido y daños en la superficie, en relación con la pérdida de la epidermis/dermis ${ }^{(14)}$.

Con relación al indicador exudado, en el presente estudio se identificó un compromiso leve de este indicador, que es equivalente a la presencia de exudado seroso. Estos datos están de acuerdo con otro estudio que encontró mitad de las lesiones investigadas con la presencia del exudado seroso $^{(14)}$.

El compromiso leve del indicador hidratación se refiere a una zona perilesional seca o resecada. La piel seca produce una sensación especial al tacto y se puede encontrar en las personas mayores y con algunas enfermedades dermatológicas crónicas, como es el caso de la UV ${ }^{(13)}$.

El compromiso leve relativo al indicador prurito se puede explicar por la dermatitis por estasis, causada por la interrupción del flujo venoso, lo que provoca la ruptura de las estructuras de la piel y aumenta el riesgo de infección local. Otros factores también pueden provocar la sensación de picazón, que son: lipodermatoesclerosis, eczema, sequedad en la región perilesional, acumulación de hemosiderina ${ }^{(15,18)}$.

Finalmente, una piel fina y lisa en la zona alrededor de la lesión, evidenciada por el comprometimiento leve para el indicador textura, se observa con frecuencia en las personas de edad y en la presencia de edema ${ }^{(13)}$. 


\section{CONCLUSIÓN}

Teniendo en cuenta los resultados que se presentan y la importancia que tienen en la orientación de una mejor atención del personal de enfermería, se considera indispensable la utilización de los resultados de enfermería de la NOC en la práctica clínica, pues permite el examen de las diversas características de la piel con el uso de indicadores operacionales. Por lo tanto, es esencial la planificación adecuada ante los datos visualizados, lo que proporciona una actuación más eficaz con relación a los indicadores mencionados como comprometidos.

\section{REFERENCIAS}

1 Brasil. Secretaria de Políticas de Saúde. Departamento de Atenção Básica. Manual de condutas para úlceras neurotróficas e traumáticas. Brasília (DF): Ministério da Saúde; 2002.

2 Eklof B, Rutherford RB, Bergan JJ, Carpentier PH, Gloviczki P, Kistner RL, et al. Revision of the CEAP classification for chronic venous disorders: consensus statement. J Vasc Surg. 2004;40:1248-52.

3 Carmo SS, Castro CD, Rios VS, Sarquis MGA. Atualidades na assistência de enfermagem a portadores de úlcera venosa. Rev Eletrônica Enferm [serie en internet]. 2007 [acceso 14 Nov 2012];9(2):[cerca de 12 p.]. Disponible en: http://www.fen.ufg.br/fen revista/v9/n2/pdf/v9n2a17.pdf

4 Maffei FHA. Insuficiência venosa crônica: conceito, prevalência etiopatogênia e fisiopatologia. In: Maffei FHA, Lastória S, Yoshida WB, Rollo HA, Giannini M, Moura Regina. Doenças vasculares periféricas. $4^{a}$ ed. Rio de janeiro: Guanabara Koogan; 2008. p. 1797-1803.

5 Maffei FHA. Diagnóstico clínico de doenças venosas periféricas. In: Maffei FHA, Lastória S, Yoshida WB, Rollo HA, Giannini M, Moura R. Doenças vasculares periféricas. 4aㅡ ed. Rio de janeiro: Guanabara Koogan; 2008. p. 491-502.

6 Abbade LPF, Lastória S. Abordagem de pacientes com úlcera da perna de etiologia venosa. An Bras Dermatol [serie en internet]. 2006 [acceso 10 Oct 2012];81(6): [cerca de 14 p.]. Disponible en: http://www.scielo.br/pdf/abd/v81n6/v81n06a02.pdf

7 Garbin LM, Rodrigues CC, Rossi LA, Carvalho EC. Classificação de resultados de enfermagem (NOC): identificação da produção científica relacionada. Rev Gaúcha Enferm [serie en internet]. 2009 [acceso 14 Nov 2012];30(3): [cerca de 8 p.]. Disponible http://seer.ufrgs.br/RevistaGauchadeEnfermagem/article/view/8216\%20/6971

8 Santos FAAS, Melo RP, Lopes MVO. Characterization of health status with regard to tissue integrity and tissue perfusion in patients with venous ulcers according to the nursing outcomes classification. J Vasc Nurs. [serie en internet]. 2010 [acceso 10 Oct 2012];28(1): [cerca de 7 p.]. http://www.sciencedirect.com/science/article/pii/S1062030309001277

9 Moorhead S, Johnson M, Maas ML, Swanson E. Classificação dos resultados de enfermagem (NOC). 4를 ed. Rio de Janeiro: Elsevier; 2010.

10 Santos FAAS. Construção de definições conceituais e operacionais do resultado Integridade Tissular aplicado a úlceras venosas [tesis]. Fortaleza: Universidade Federal do Ceará; 2011.

11 Arango HG. Bioestatística teórica e computacional. $3^{\mathrm{a}}$ ed. Rio de Janeiro: Guanabara Koogan; 2009.

12 Silva FAA, Moreira TMM. Características sociodemográficas e clínicas de clientes com úlcera venosa de perna. Rev Enferm UERJ. 2011;19(3):468-72. 
13 Porto CC. Exame clínico: bases para a prática médica. $6^{\underline{a}}$ ed. Rio de Janeiro: Guanabara Koogan; 2008.

14 Benevides JP, Coutinho JFV, Santos MCL, Oliveira MJA, Vasconcelos FF. Avaliação clínica de úlceras de perna em idosos. Rev Rene. [serie en internet]. 2012 [acceso 20 Nov 2012];13(2): [cerca de 9 p.]. Disponible en: http://www.revistarene.ufc.br/revista/index.php/revista/article/view/213/pdf

15 Bersusa AAS, Lages JS. Integridade da pele prejudicada: identificando e diferenciando uma úlcera arterial e uma venosa. Ciênc Cuid Saúde. [serie en internet]. 2004 [acceso 20 Nov 2012];3(1): [cerca de 12 p.]. Disponible en: http://periodicos.uem.br/ojs/index.php/CiencCuidSaude/article/view/5521/3511.

16 Costa IKF, Melo GSM, Farias TYA, Tourinho FSV, Enders BC, Torres GV, et al. Influence of pain on daily life of people with venous ulcers: evidence-based practice. Journal of Nursing UFPE on line. [serie en internet]. 2011 [acceso 14 Nov 2012];5(2):[cerca $\quad$ de $\quad 8 \quad 10$ p.] Disponible http://www.revista.ufpe.br/revistaenfermagem/index.php/revista/article/view/1738

17 Wong IKY, Andriessen A, Lee DTF, Thompson D, Wong LY, Chao DVK et al. Randomized controlled trial comparing treatment outcome of two compression bandaging systems and standard care without compression in patients with venous leg ulcers. J Vasc Surg. [serie en internet]. 2012 [acceso 14 Sep 2012];55(5). Disponible en: http://onlinelibrary.wiley.com/doi/10.1111/j.1468-3083.2011.04327.x/pdf

18 Carrasco JMH, Dumont EL, Gálvez FR, Gutiérrez MG, Montesinos PS, García MAG. Un antes y un después: del riesgo o deterioro de la integridad cutánea a la integridad tisular. Gerokomos. 2008;19(3):153-158. 